\title{
Effect of the preparation method on the drug loading of alginate-chitosan microspheres
}

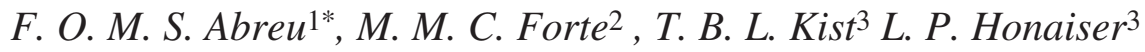 \\ ${ }^{1}$ Laboratory of Biopolymers/ Analytical and Physical-Chemistry Department, Federal University of Ceará-UFC, \\ Fortaleza-CE, Brazil \\ ${ }^{2}$ Laboratory of Polymeric Materials/Engineering School, PO Box 15.010, Federal University of Rio Grande do Sul \\ (UFRGS), Porto Alegre-RS, Brazil \\ ${ }^{3}$ Laboratory of Analytical Methods/Bioscience Institute, Federal University of Rio Grande do Sul-UFRGS, Porto \\ Alegre-RS, Brazil
}

Received 13 January 2010; accepted in revised form 4 March 2010

\begin{abstract}
Alginate-chitosan (ALG-CHI) microspheres obtained by polyelectrolyte complexation are pH-sensitive, biocompatible and adhesive, and are excellent candidates for the delivery of drugs, proteins and peptides in the human body. A wide variety of methods for the production of these polymeric complexes has been provided. The water-in-oil emulsion is a complex production method, but generally enhances the control of particle size and particle size distribution of the microspheres, extremely necessary for obtaining repeatable controlled release behavior. In this work, a novel and facile water-in-oil emulsion method for the ALG-CHI polyelectrolyte complexes is discussed. The method proposed produced ALG-CHI microspheres with improved morphology and enhanced drug loading in comparison with the aqueous medium method. The drug loading in the water-in-oil emulsion was over $30 \%$ higher than in the aqueous medium, an indication that the new method proposed the common drug leaching during the microspheres' preparation is avoided, being an interesting alternative to encapsulate drugs of hydrophilic nature.
\end{abstract}

Keywords: biodegradable polymers, polymer gels, microspheres, chitosan, drug loading

\section{Introduction}

Alginate-chitosan hydrogels (ALG-CHI) have been proposed as drug delivery system in the past decade, due to their attractive combination of $\mathrm{pH}$ sensitivity, bio-compatibility and adhesiveness, requiring relative mild gelation conditions for the network formation [1]. A great deal of processes was developed for these hydrogels' production in the last few years [2]. One of the limitations of these hydrogels is the drug leaching during their preparation [3] which can be reduced by controlling the reactions conditions [4-7]. In a previous work [8] several ALG-CHI formulations were statistically investigated in aqueous medium in order to modulate and control the polyelectrolyte complexation and subsequently the hydrogel properties. In another report, the influence of the introduction of some carboxylic groups on chitosan was studied and the effects on the formation of polymer complexes with ALG at pH 4 and 6 [9]. In both reports, microspheres were obtained with high yield, low particle size and desirable swelling ability required in the intestinal media for promising drug release. Emulsion methods have been proposed in order to increase the encapsulation efficiency of drugs using low water solubility polymers [10-12].

Gel bead system based on calcium alginate and chitosan were successfully produced in oil-in-water 
emulsion for oral delivery of allyl isothiocyanate [10]. Hydrogel microspheres of chitosan crosslinked with glutaraldehyde with uniform-size have been produced by a membrane emulsification technique [11], and a water-in-oil (W/O) emulsion coalescence technique was proposed for the production of CHI particles using vegetable oil [12]. Both methods generated microspheres with enhanced control of particle size and particle size distribution, which are important for repeatable controlled release behavior $[11,12]$. Despite some different water-inoil methods have been used to produce microspheres [13-16], they have not been utilized for the preparation of ALG-CHI microspheres to enhance the encapsulation of water-soluble drugs.

Ionic and covalent crosslinkers can be added to the ALG-CHI system for improving the properties. Calcium chloride is frequently used as an ionic crosslinker in ALG-CHI systems [7-9, 11] causing a reduction in the hydrogel porosity [17]. Among the covalent crosslinkers, there are some reports for chitosan nanoparticles crosslinked with glutaraldeyde $[13,18]$ and genipin $[19,20]$. Genipin is a natural covalent crosslinker that presents very low toxicity in comparison with conventional crosslinkers and can present promising results in the reinforcement of ALG-CHI based microspheres [20].

In this work, a novel W/O emulsion method was designed for ALG-CHI microspheres' preparation using two types of crosslinker, calcium ions and genipin, and two types of surfactant agents with a view to increase the drug loading of some model drugs. The properties of the ALG-CHI particles produced in emulsion by different methods were compared with those obtained by the aqueous method.

\section{Experimental}

\subsection{Materials}

Alginic acid sodium salt $(\sim 250 \mathrm{mPa} \cdot \mathrm{s}$ viscosity at $25^{\circ} \mathrm{C} 1 \mathrm{wt} \%, 64.4 \mathrm{kDa}$ ) and polyvinyl pirrolidone (40 kDa) was purchased from Sigma. Low molecular weight Chitosan (90\% deacetylated, $6.68 \mathrm{kDa}$ ) was purchased from Aldrich. Calcium chloride and polyvinyl alcohol (86.5-89.5\% hydrolyzed, 30$70 \mathrm{kDa}$ ) were purchased from Synth. Genipin was purchased from Challenge Bioproducts. Fluorescein salt was supplied by Synth, lisinopril and fluo- rescein isothiocianate were supplied by Sigma. All reagents were analytical grades and were used as received.

\subsection{Microspheres preparation}

ALG-CHI microspheres were produced in W/O emulsion, testing two types of surfactant and two types of crosslinker, resulting in four different formulations in emulsion. Also ALG-CHI microspheres were produced in aqueous medium, as described in earlier work [8], for comparison of properties. Polymer complexes were prepared using appropriate proportions, in order to obtain hydrogels with polymer ratio ALG:CHI $=35: 65$, condition previously optimized in previous articles $[8,9]$. The model drugs were added using a mass ratio Polymer:Drug of 5:1, condition optimized prior the design. Six formulations were produced in triplicate, the conditions are shown in Table 1.

Aqueous solution of ALG $(1 \% \mathrm{w} / \mathrm{v})$ were prepared and diluted to a final concentration of $0.2 \% \mathrm{w} / \mathrm{v}$ using distilled water. $\mathrm{CHI}$ solution $(1 \% \mathrm{w} / \mathrm{v})$ were dissolved in an acetic acid solution with $\mathrm{pH}=3$, and further diluted to $(0.2 \% \mathrm{w} / \mathrm{v})$ with distilled water. ALG-CHI-Ca ${ }^{+2}$ microspheres were prepared by placing the solution of $\mathrm{CHI} 0.2 \%$ and ALG $0.2 \%$ in separate tubes and adding the $2 \mathrm{mM}$ $\mathrm{CaCl}_{2}$ solution into the tube with $\mathrm{CHI}$ solution and homogenized. The surfactant powder was added in each tube in a $1.5 \% \mathrm{w} / \mathrm{v}$ concentration and homogenized in an ultrasonic bath for $25 \mathrm{~min}$. Both tubes were carefully added to a vessel containing mineral oil in a volume ratio 6:1 mineral oil: aqueous phase. The mixture was vigorously sonicated with an ultrasonic probe for 3 min producing a stable emulsion, and then replaced in the ultrasonic bath for additional $20 \mathrm{~min}$. The emulsion was centrifuged at $3500 \mathrm{rpm}$ for $30 \mathrm{~min}$ for aqueous and oil-phase

Table 1. Formulations used for production of ALG-CHI microparticles in W/O emulsion and aqueous method

\begin{tabular}{|l|c|c|c|}
\hline Formulations & $\begin{array}{c}\text { Preparation } \\
\text { method }\end{array}$ & $\begin{array}{c}\text { Surfactant } \\
\text { type }\end{array}$ & $\begin{array}{c}\text { Crosslinker } \\
\text { type }\end{array}$ \\
\hline 1-AQ/Ca & Aqueous & - & $\mathrm{CaCl}_{2}$ \\
\hline 2-AQ/gen & Aqueous & - & $\mathrm{Genipin}$ \\
\hline 3-EM/PVA/Ca & Emulsion & PVA & $\mathrm{CaCl}_{2}$ \\
\hline 4-EM/PVA/Gen & Emulsion & PVA & $\mathrm{Genipin}$ \\
\hline 5-EM/PVP/Ca & Emulsion & PVP & $\mathrm{CaCl}_{2}$ \\
\hline 6-EM/PVP/Gen & Emulsion & PVP & $\mathrm{Genipin}$ \\
\hline
\end{tabular}



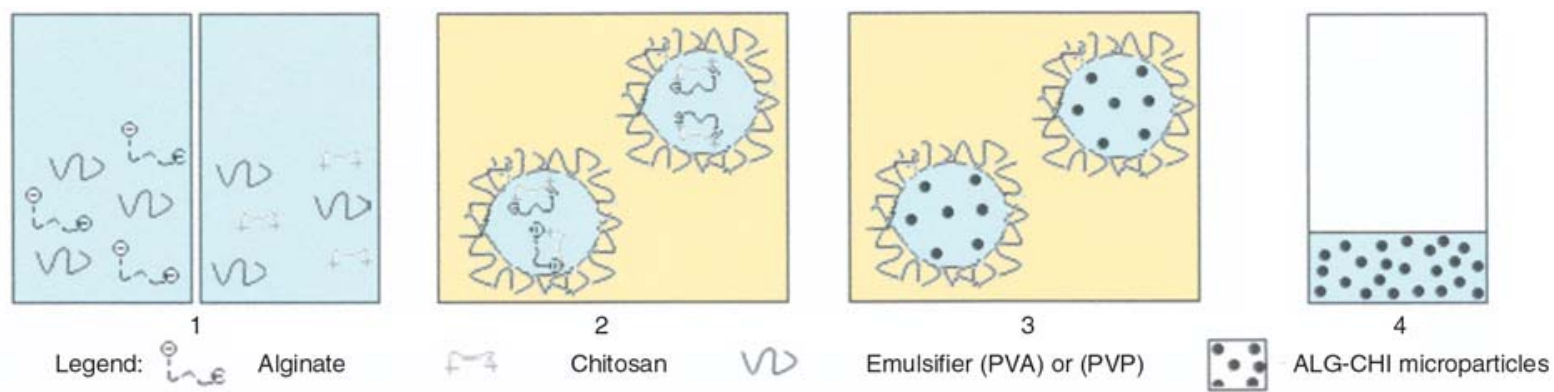

Figure 1. Schematic representation of ALG-CHI particles preparation by W/O emulsion method. Step 1: Aqueous solution having ALG, CHI and surfactant; Step 2: Stable water-in-oil emulsion; Step 3: ALG-CHI particles in the water-in-oil emulsion micelles; Step 4: ALG-CHI particles separated in the aqueous phase.

separation, than the aqueous-phase was again centrifuged and the solid obtained was lyophilized. The preparation of the microspheres ALG-CHI-genipin was similar; the genipin solution $(0.1 \% \mathrm{w} / \mathrm{w})$ was added in the tube containing ALG, and there was no addition of $\mathrm{CaCl}_{2}$ in the system. ALG-CHI microspheres loaded with the models drugs fluorescein or lisinopril labeled with FITC were prepared by adding the model drugs in the tube containing the $\mathrm{CHI}$ solution and homogenized. Figure 1 shows a schematic representation of the components in all steps of the ALG-CHI nanoparticles preparation by the water-in-oil method.

\subsection{Microspheres characterization}

Hydrogel samples were dried and then sputtercoated with gold for Scanning Electron Microscope (SEM) characterization in a Jeol JSM 5800 microscope, using an acceleration voltage of $5 \mathrm{kV}$. The morphology was investigated through Fluorescence Optical microscope (OM) (Leitz Wetzlar). The loading and the encapsulation efficiency were determined by UV/VIS spectroscopy, at 489 and $499 \mathrm{~nm}$, in a MICRONAL-Brazil spectrometer (model B582) as following: a $10 \mathrm{mg}$ sample was crushed in ethanol and its concentration was calculated using a calibration curve obtained from samples of pure fluorescein at $489 \mathrm{~nm}$ and lisinopril labeled with fluorescein isothiocianate (FITC) at $499 \mathrm{~nm}$ at certain concentration range. All analysis was replicated twice.

The encapsulation efficiency $(E E)$ was evaluated for fluorescein as model drug and for lisinopril labeled with FITC as shown by Equation (1):

$$
\% E E=\frac{M}{M_{0}} \cdot 100
$$

where $M$ is the amount of drug in loaded sample, as determined from the calibration curve and $M_{0}$ is the initial drug amount added to the complex.

\section{Results and discussion}

ALG-CHI microspheres were prepared from $0.2 \mathrm{wt} \%$ polymer solution having the polymer mass ratio $\mathrm{ALG} / \mathrm{CHI}=35 / 65$. These conditions were chosen due to good particle stability and properties reported previously $[8,9]$, where hydrogels presented higher yield and lower swelling degree. In a previous study, it was seen that CHI based microspheres prepared at aqueous medium showed low encapsulation efficiency of hydrophilic drugs (unpublished results). This could be related to the drug highly hydrophilic character, which present stronger interaction between drug-solvent (water) than the electrostatic interactions between drugmicrospheres. In this work, microspheres with controlled morphology were produced by emulsion method and by aqueous method, using two types of crosslinker and surfactant, focusing in differences in the morphology and drug distribution pattern, aiming at encapsulation efficiency optimization.

\subsection{Morphology}

The morphology of the ALG-CHI microspheres was observed through SEM, as shown in Figure 2. The ALG-CHI hydrogel particles showed spherical shape and the average particle size and particles size distribution varied according to the preparation method, in aqueous medium or W/O emulsion, and to the crosslinker type, genipin or $\mathrm{CaCl}_{2}$. In general, there was an increase in the regularity of the particles shape when genipin was used as crosslinker agent, as revealed by the SEM micrographs Fig- 


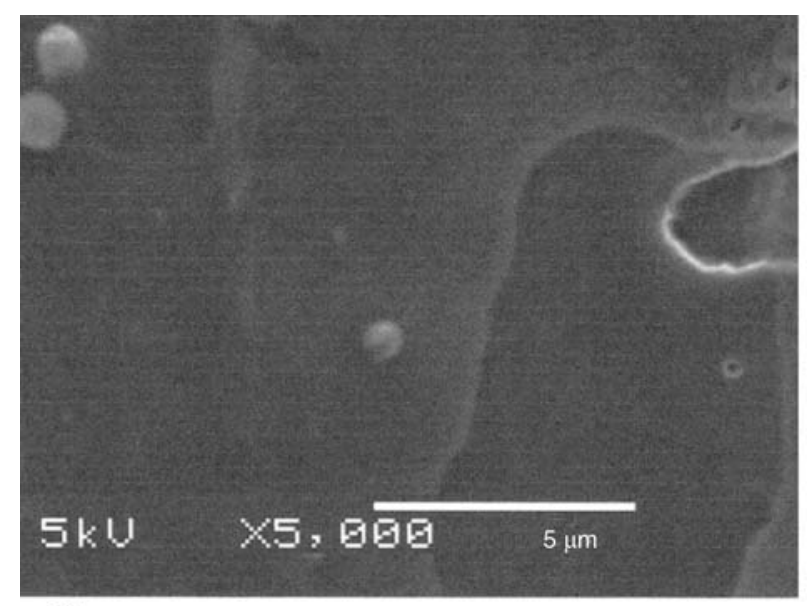

a)

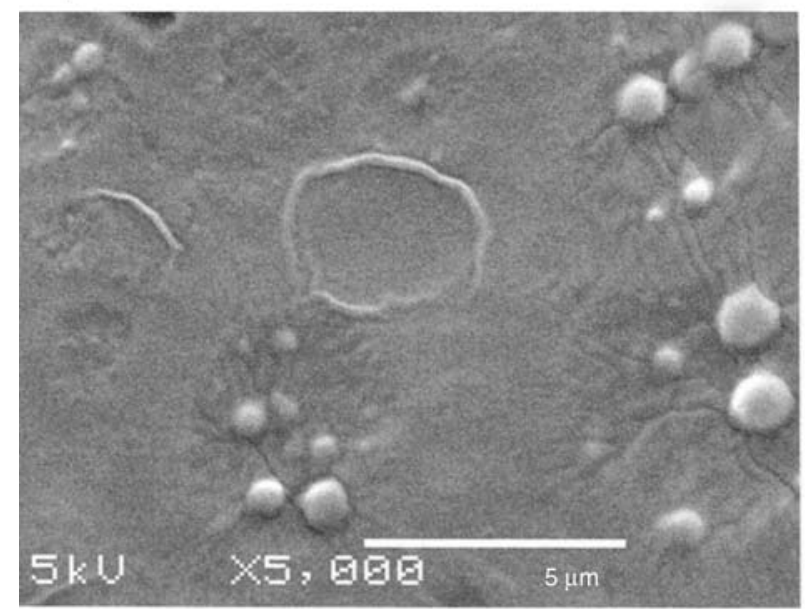

c)

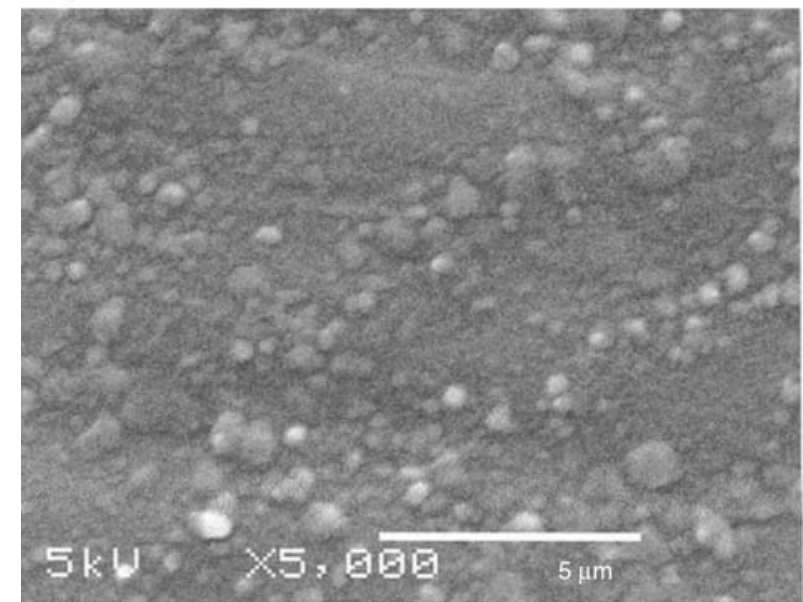

e)

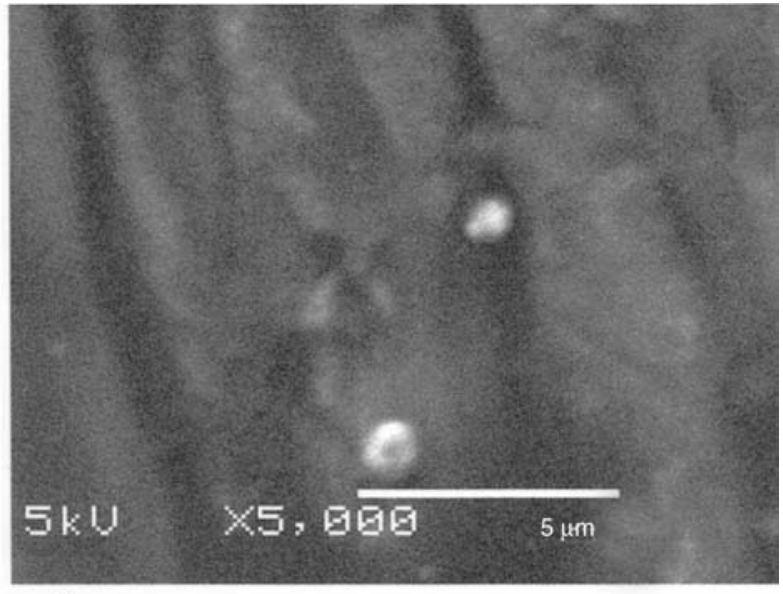

b)

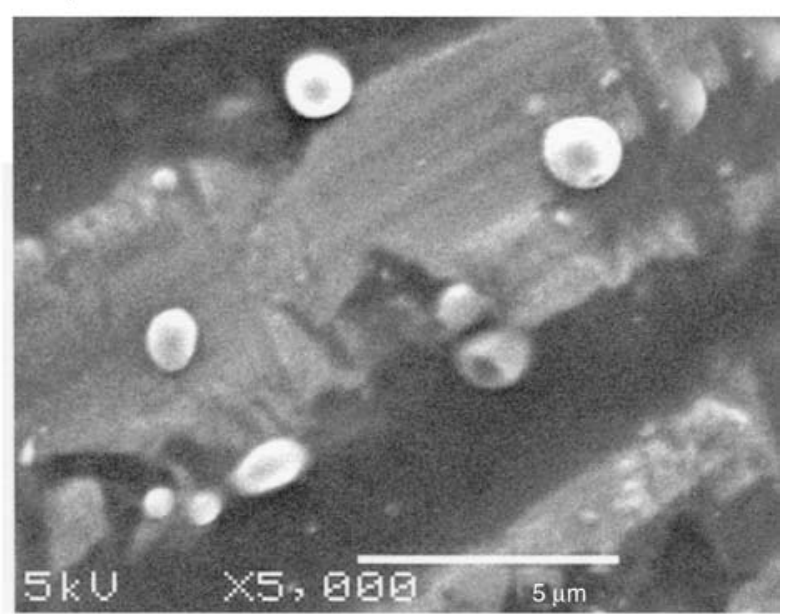

d)

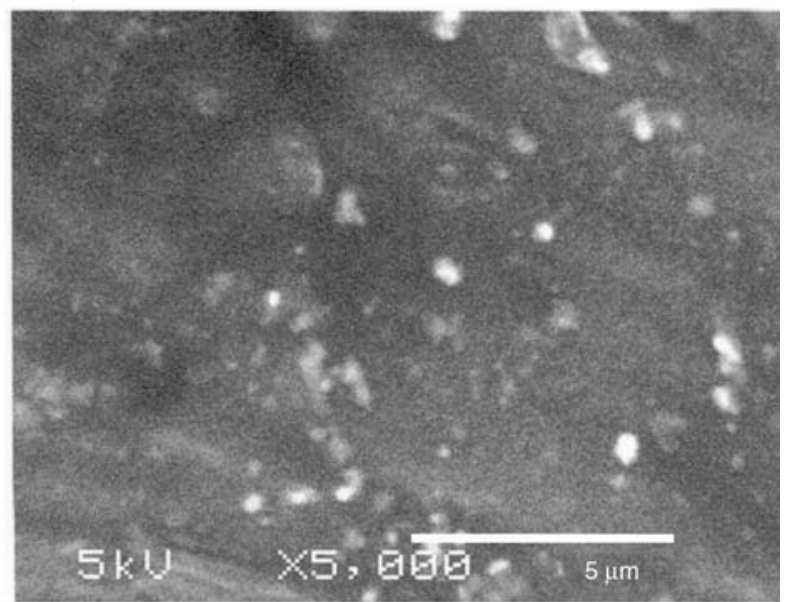

Figure 2. SEM micrograph images of ALG-CHI microparticles obtained using $\mathrm{CaCl}_{2}$ as croslinker: by aqueous method with $\mathrm{CaCl}_{2}$ (a) and with genipin (b), and by W/O emulsion method using PVA with genipin (c) and with $\mathrm{CaCl}_{2}$ (d) and using PVP with Genipin (e) and with $\mathrm{CaCl}_{2}$ (f)

ure $2 \mathrm{a}, 2 \mathrm{c}$ and $2 \mathrm{e}$. ALG-CHI particles produced in W/O emulsion with PVA using both crosslinker agents presented a more spherical shape and regular structure, revealed by the SEM micrographs Figure $2 \mathrm{c}$ and $2 \mathrm{~d}$.
Figure 3 shows OM micrographs of ALG-CHI microspheres obtained by aqueous and emulsion method. Microspheres produced through the former method were more irregular in shape and there was a tendency to form agglomerates (Figure 3a 


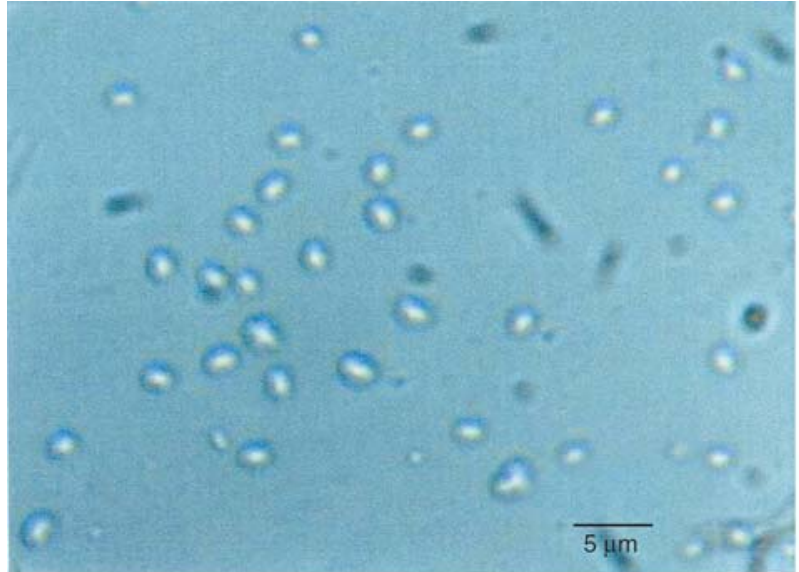

a)

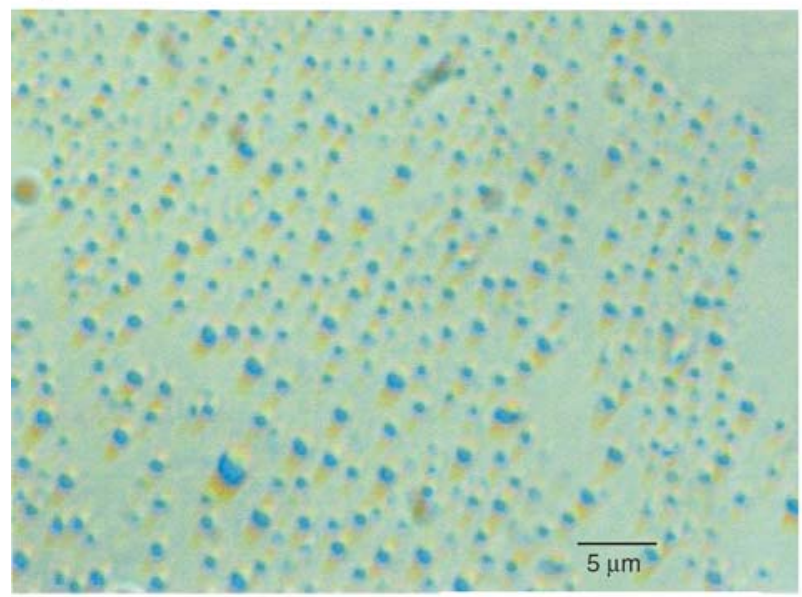

c)

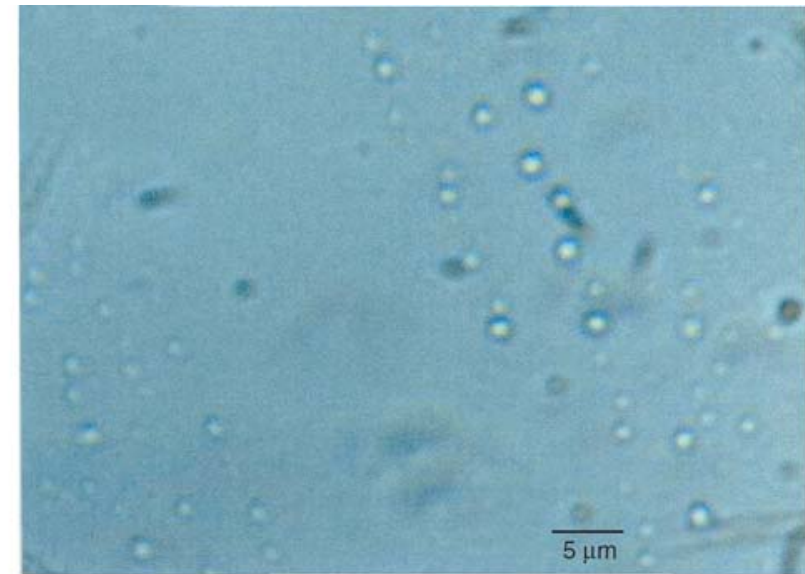

b)

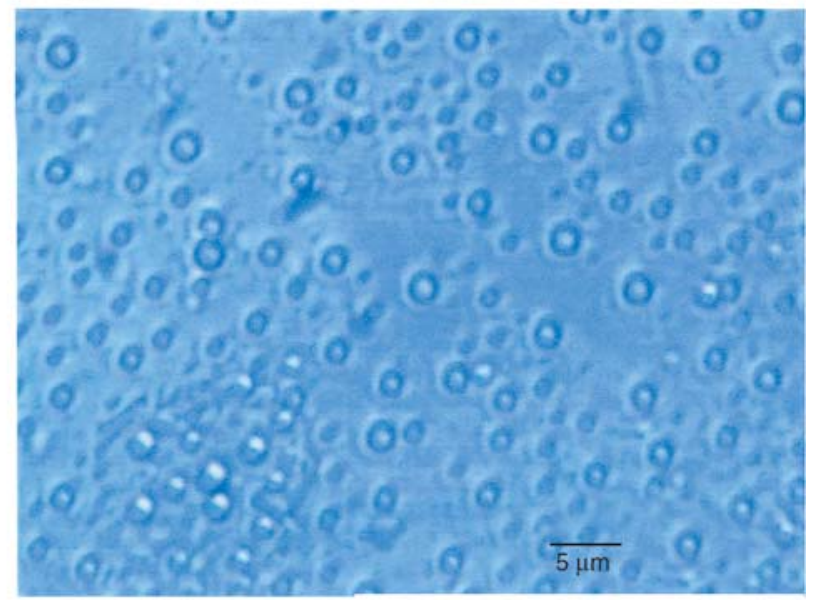

d)

Figure 3. $\mathrm{MO}$ micrograph images of ALG-CHI microparticles obtained in aqueous medium with genipin (a) and with $\mathrm{CaCl}_{2}$ (b), and by W/O emulsion method using PVA with genipin (c) and PVP with $\mathrm{CaCl}_{2}(\mathrm{~d})$

and $3 b)$. The particle agglomeration in the aqueous method is possibly caused by the formation of inter-aggregate complexes between the ALG carboxylic chains in the hydrogel induced by $\mathrm{Ca}^{+2}$. This is in accordance with Zhu et al. [21] results, in which the agglomeration effect was observed for carboxymethyl-chitosan aqueous solutions in presence of metallic ions. This agglomeration that occurs simply by electrostatic interactions between the ions and the polymers may lead to the formation of microparticles with irregular morphology and higher polydispersity. On the other hand, microspheres prepared through the emulsion method presented uniform size with self-avoiding particles (Figure $3 \mathrm{c}$ and $3 \mathrm{~d}$ ), being potentially more adequate for drug delivery. The emulsion method, due to the ALG-CHI particles arrangement inside the droplets, may prevent the formation of inter-aggregated complex caused by electrostatic interactions. Studies regarding the preparation of microparticles by emulsion were conducted by others; Kofuji et al. [12] reported chitosan microparticles chelated by metallic produced by emulsion technique with irregular morphology and rough surface; on the other hand, Wang et al. [13] obtained chitosan microparticles with excellent size control and smooth surface by a innovative membrane emulsification technique. Table 2 shows the average particle size of ALG-CHI microspheres. The average particle size of the ALG-CHI microspheres corresponds to the average diameter measured on fifty

Table 2. Encapsulation efficiency and average particle size of ALG-CHI microparticles obtained in different formulations

\begin{tabular}{|l|c|c|c|}
\hline \multirow{2}{*}{ Formulations } & \multicolumn{2}{|c|}{ Encapsulation efficiency [\%] } & Particle size \\
\cline { 2 - 3 } & Fluorescein & Lisinopril & $1.3 \pm 0.6$ \\
\hline 1-AQ/Ca & $38 \pm 15$ & $45 \pm 12$ & $1.6 \pm 0.9$ \\
\hline 2-AQ/gen & $50 \pm 12$ & $60 \pm 9$ & $0.5 \pm 0.3$ \\
\hline 3-EM/PVA/Ca & $83 \pm 10$ & $80 \pm 8$ & $0.8 \pm 1$ \\
\hline 4-EM/PVA/Gen & $77 \pm 8$ & $86 \pm 7$ & $0.7 \pm 0.3$ \\
\hline 5-EM/PVP/Ca & $78 \pm 4$ & $74 \pm 9$ & $0.6 \pm 0.2$ \\
\hline 6-EM/PVP/Gen & $29 \pm 16$ & $37 \pm 10$ & \\
\hline
\end{tabular}


particles from each batch. It can be seen from the average diameter values that the particles size varied according to the preparation method and composition used. Microparticles produced by the W/O emulsion method were smaller having narrower particle size distribution than those produced by the aqueous method. Chitosan microparticles chelated by a metal ion obtained by emulsion coalescence technique in vegetal oil presented uniform size with low polidispersity [12]. Some data on emulsion methods report particles having broad size range, due to the use of high-speed blenders and high pressure homogenizers which involves mechanical shear force to reduce the size of the emulsion droplets and forms polydisperse particles [15]. However, the formulations produced by emulsion method with PVP as surfactant presented lower polydispersity than those produced with PVA and equivalent polydispersity to those produced by aqueous medium method. PVP perhaps exhibits better stabilizing effect, where the adsorption of the polymer in the droplets surface avoids the coagulation and consequently the agglomeration of droplets. On the other hand, PVA presents higher molecular weight than PVP (30-70 and $40 \mathrm{kDa}$, respectively) and higher affinity to the ALG-CHI polymers. In this sense, different segments of a single PVA macromolecule may absorb simultaneously on two or more droplets, leading to an attractive force which results in droplets aggregation, with consequently higher particle size and high polidispersity.

\subsection{Encapsulation efficiency}

Using $2 \%$ of loading of the drug models, the encapsulation efficiency was determined using a calibration curve in a UV-visible spectrometer, as described earlier. The calibration curves for fluorescein and for lisinopril labeled with FITC are in Figure 4. The correlation between absorbance and concentration for fluorescein and lisinopril are given respectively by the Equations (2) and (3):

$$
\begin{array}{ll}
y=0.035 x-0.004, & R^{2}=0.998 \\
y=0.0204 x-0.0715, & R^{2}=0.992
\end{array}
$$

The curves presented excellent linearity for low and high drug concentration, with a correlation factor $R^{2}>0.99$. The model drug's concentrations were

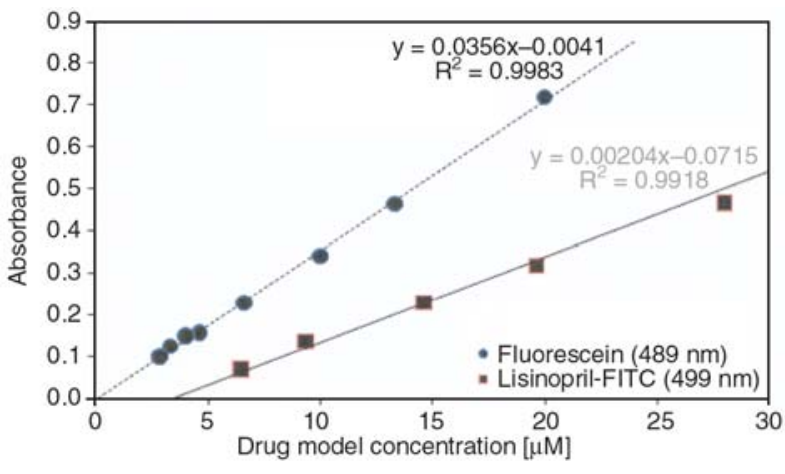

Figure 4. Calibration curve for fluorescein and lisionpril labeled with Isothiocianate

calculated using Equations (2) and (3) and the encapsulation efficiency values for each system produced were calculated using Equation (1), as shown in Table 2. Comparing the encapsulation efficiency $(E E)$ values is observed that particles produced in aqueous medium presented relatively low $E E$ in comparison with the majority of the $E E$ values of the microspheres obtained by W/O emulsion method, which successfully entrapped over $70 \%$ of lisinopril and fluorescein. The $E E$ of lisinopril-FITC was higher or equivalent than fluorescein, probably due to the lower hydrophilic character which may cause lower drug leaching. Genipin was more effective as crosslinker than $\mathrm{Ca}^{+2}$ for ALG-CHI microparticles loaded with lisinopril produced by aqueous method and also by W/O emulsion with PVA. In particular, there was a notable increase of $15 \%$ in the encapsulation efficiency of lisinopril when genipin was used instead of $\mathrm{CaCl}_{2}$ in the hydrogel preparation through the aqueous method. As previously reported [19], controlled release rates of indomethacin from ALG$\mathrm{CHI}$ particles were achieved by increasing the genipin content in the hydrogel due to a higher crosslinking density. In fact, the genipin content can be manipulated in order to control the chitosan crosslink density [20]. On the other hand, when $\mathrm{CaCl}_{2}$ was used as crosslinking agent using the W/O emulsion method the surfactant type did not have strong influence in the efficiency of both model drugs, and the formulations presented high $E E$ values for both drugs. An analysis of the surfactant effect in the $E E$ shows that it with PVA it was achieved the highest values of encapsulation efficiency for both drugs and using both types of crosslinker. Despite the fact that PVA has formed particles with higher polidispersity, depending 
upon the application of the delivery system, it might be desirable to achieve high $E E$ values with a decrease of the control of the size.

Figure 5 shows the lisinopril-FITC encapsulation and distribution within the particles investigated by Fluorescence Optical microcopy (FOM). It is evidenced in the lisinopril-FITC-ALG-CHI microspheres micrographs that the distribution pattern varied according to the preparation method and crosslinker type. In aqueous medium, the drug dispersion was favored by replacing $\mathrm{CaCl}_{2}$ with genipin (Figure 5a and 5b, respectively) also corroborated by the $E E$ values. In Figure $5 \mathrm{c}$ and $5 \mathrm{~d}$ the homogeneous dispersion of the drug is shown inside the ALG-CHI microspheres produced through

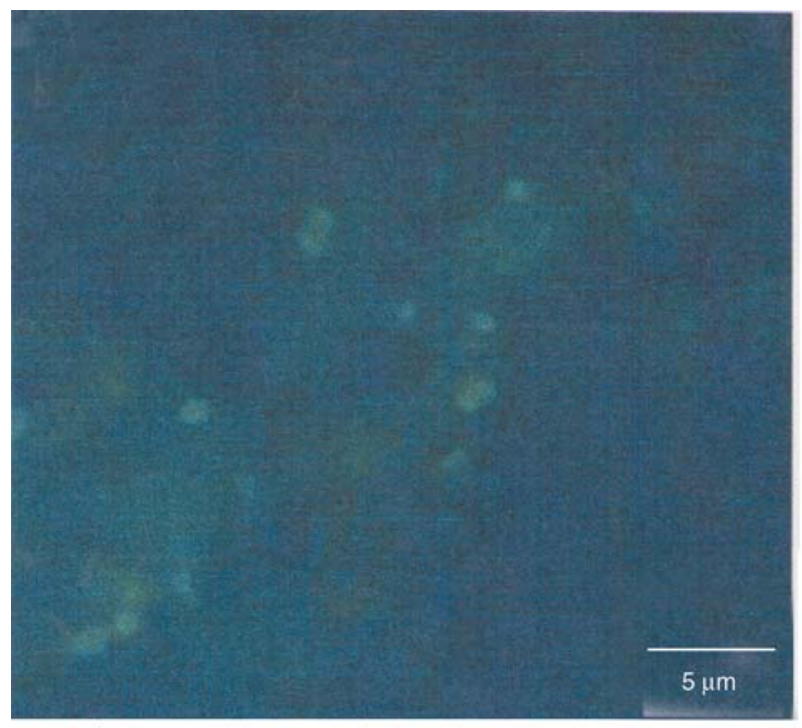

a)

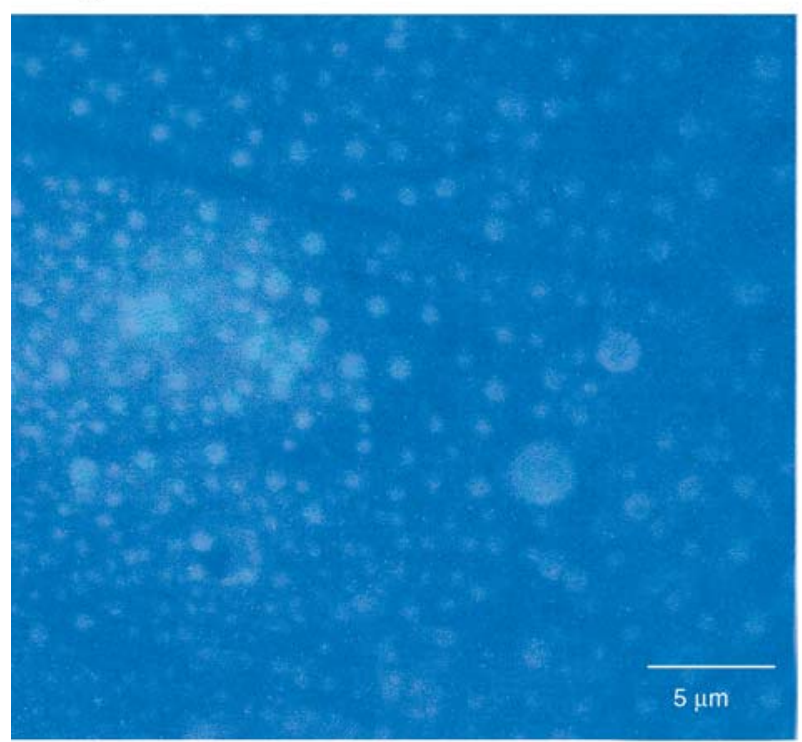

c) the W/O emulsion method. Particularly, the surfactant seems to affect the encapsulation efficiency of ALG-CHI particles crosslinked with genipin. The reported mechanism for genipin-chitosan crosslinking reaction is a nucleophilic attack of chitosan amino groups in the dihydropiran ring of genipin [20]. A high loss of hydrogen ions from protonated amino groups to the carboxylic groups favors the nucleophilicity of amino groups, which in turn may increase the CHI crosslinking density [20]. In the case of the microspheres here reported, the residual carboxylic groups $(25 \%)$ in the PVA molecules may favor the chitosan deprotonation, which facilitates the amino nucleophilic attack, increases the crosslinking density and consequently the encapsu-

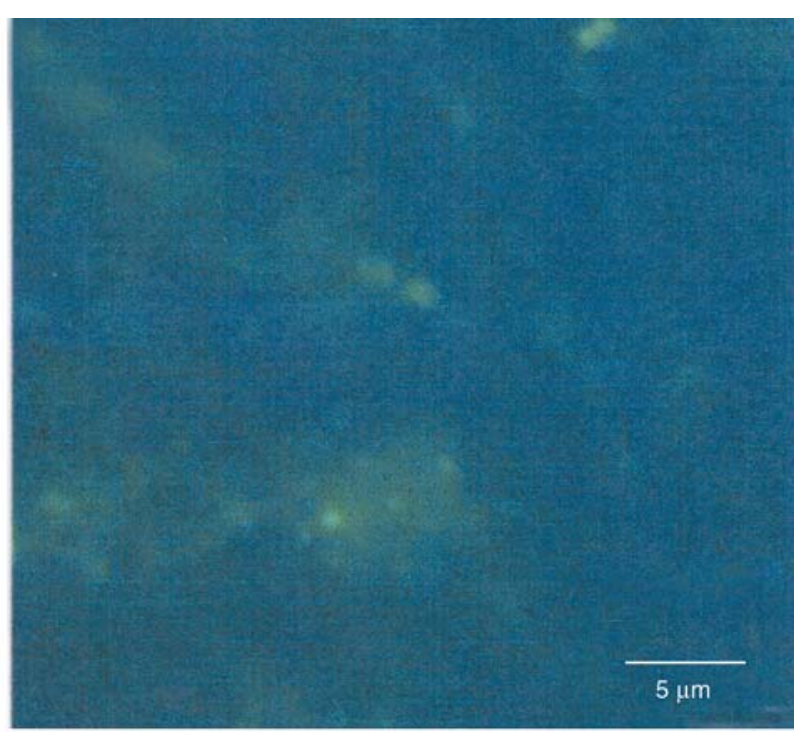

b)

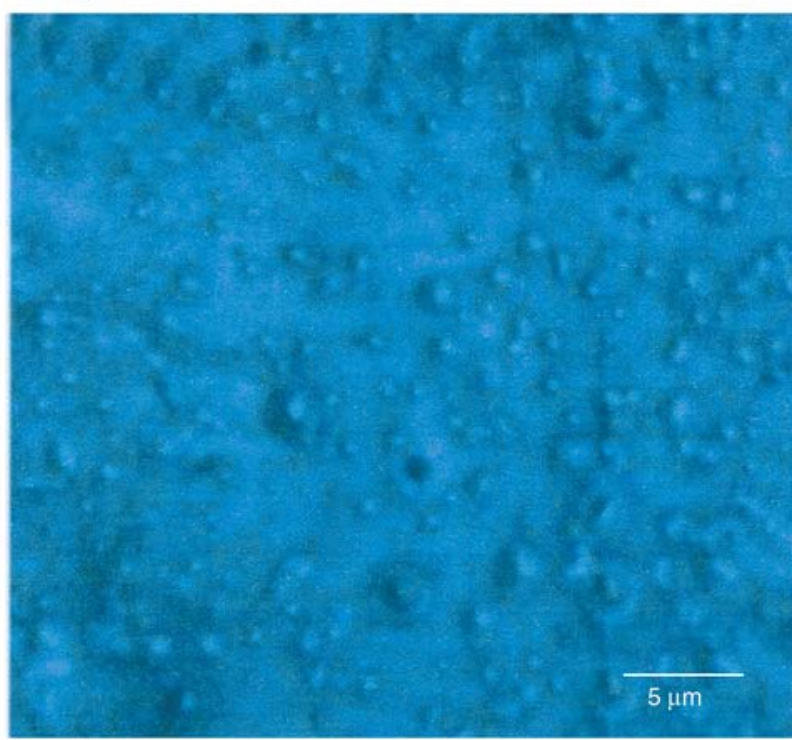

d)

Figure 5. FOM micrograph images for drug distribution pattern. ALG-CHI microparticles obtained in aqueous medium with $\mathrm{CaCl}_{2}$ (a) and with genipin (b), and by W/O emulsion method using PVA with $\mathrm{CaCl}_{2}$ (c) and PVP with genipin (c) 
lation efficiency ( $85 \%$ for the system EM/PVA/ Gen loaded with lisinopril). On the other hand, in the case of the ionic crosslinker (calcium chloride), PVP and PVA were a good surfactant for the W/O system and the average $E E$ was around $75 \%$.

In summary, ALG-CHI microspheres produced in W/O emulsion with PVA as surfactant using both types of crosslinker presented highly spherical particles with acceptable size distribution for a variety of delivery systems. On the other hand, the emulsion system ALG-CHI-Ca ${ }^{+2}$ using PVP as crosslinker presented spherical particles with lower polidispersity, desirable for some specific applications. In addition, those particles formed self-avoided domains, avoiding aggregation. Moreover, these particles presented higher encapsulation efficiency reaching values up to $75 \%$ than those produced in aqueous medium.

\section{Conclusions}

Different methods for production of ALG-CHI microspheres were tested in order to increase the encapsulation efficiency and optimize the morphology. Regarding the crosslinker type, genipin increased significantly the $E E$ of microspheres produced in aqueous medium. It was observed that the emulsion method in general was more efficient to encapsulate the model drugs than the aqueous method. Moreover, ALG-CHI microspheres produced in emulsion using PVA as surfactant presented highly spherical particles with acceptable size distribution, and the emulsion system ALG$\mathrm{CHI}-\mathrm{Ca}^{+2}$ using PVP as crosslinker presented lower particle size with higher size control, desirable for some specific applications. Those particles formed self-avoided domains, avoiding aggregation and higher encapsulation efficiency reaching values up to $75 \%$, indicating that the emulsion method is a promising route to encapsulate hydrophilic drugs.

\section{Acknowledgements}

The authors thank the Governmental Agencies CNPq and CAPES for the financial support to this work and to Dr. Hamid Elassari (France) for the exchange of ideas.

\section{References}

[1] Berger J., Reist M., Mayer J. M., Felt O., Peppas N. A., Gurny R.: Structure and interactions in covalently and ionically crosslinked chitosan hydrogels for biomedical applications. European Journal of Pharmaceutics and Biopharmaceutics, 57, 19-34 (2004). DOI: $\underline{10.1016 / \mathrm{S} 0939-6411(03) 00161-9}$

[2] Sinha V. R., Singla A. K., Wadhawan S., Kaushik R., Kumria R., Bansal K., Dhawan S.: Chitosan microspheres as a potential carrier for drugs. International Journal of Pharmaceutics, 274, 1-33 (2004). DOI: 10.1016/j.ijpharm.2003.12.026

[3] George M., Abraham T. E.: Polyionic hydrocolloids for the intestinal delivery of protein drugs: Alginate and chitosan- A review. Journal of Controlled Release, 114, 1-14 (2006). DOI: 10.1016/j.jconrel.2006.04.017

[4] De S., Robinson D.: Polymer relationships during preparation of chitosan-alginate and poly-l-lysinealginate nanospheres. Journal of Controlled Release, 89, 101-112 (2003). DOI: $\underline{10.1016 / \mathrm{S} 0168-3659(03) 00098-1}$

[5] Ribeiro A. J., Silva C., Ferreira D., Veiga A.: Chitosan-reinforced alginate microspheres obtained through the emulsification/internal gelation technique. European Journal of Pharmaceutical Science, 25, 3140 (2005). DOI: $10.1016 /$ j.ejps.2005.01.016

[6] Wittaya-areekul S., Kruenate J., Prahsarn E. C.: Preparation and in vitro evaluation of mucoadhesive properties of alginate/chitosan microparticles containing prednisolone. International Journal of Pharmaceutics, 312, 113-118 (2006). DOI: 10.1016/j.ijpharm.2006.01.003

[7] Alsarra I. A., Betigeri S. S., Zhang H., Evans B. A., Neau S. H.: Molecular weight and degree of deacetylation effects on lipase-loaded chitosan bead characteristics. Biomaterials, 23, 3637-3644 (2002). DOI: $10.1016 / \mathrm{S} 0142-9612(02) 00096-0$

[8] Abreu F. O. M. S., Bianchini C., Forte M. M. C., Kist T. B. L.: Influence of the composition and preparation method on the morphology and swelling behavior of alginate-chitosan hydrogels. Carbohydrate Polymers, 74, 283-289 (2008). DOI: 10.1016/j.carbpol.2008.02.017

[9] Abreu F. O. M. S., Bianchini C., Kist T. B. L., Forte M. M. C.: Preparation and properties of core-shell alginate-carboxymethylchitosan hydrogels. Polymer International, 58, 1267-1274 (2009). DOI: $\underline{10.1002 / p i .2657}$

[10] Kim W-T., Chung H., Shin I-S., Yam K. L., Chung D.: Characterization of calcium alginate and chitosantreated calcium alginate gel beads entrapping allyl isothiocyanate. Carbohydrate Polymers, 71, 566-573 (2008). DOI: 1 10.1016/j.carbpol.2007.06.028 
[11] Wang L-Y., Ma G-H., Su Z-G.: Preparation of uniform sized chitosan microspheres by membrane emulsification technique and application as a carrier of protein drug. Journal of Controlled Release, 106, 6275 (2005).

DOI: $\underline{10.1016 / j . j \text { conrel.2005.04.005 }}$

[12] Kofuji K., Qian C-J., Murata Y., Kawashima S.: Preparation of chitosan microparticles by water-invegetable oil emulsion coalescence technique. Reactive and Functional Polymers, 62, 77-83 (2005).

DOI: $10.1016 /$ j.reactfunctpolym.2004.09.002

[13] Wang L-Y., Gu Y-H., Zhou Q-Z., Ma G-H., Wan YH., Su Z-G.: Preparation and characterization of uniform-sized chitosan microspheres containing insulin by membrane emulsification and a two-step solidification process. Colloids and Surfaces B: Biointerfaces, 50, 126-135 (2006).

DOI: $\underline{10.1016 / j . c o l s u r f b .2006 .05 .006}$

[14] Zhi J., Wang Y., Lu Y., Ma J., Luo G.: In situ preparation of magnetic chitosan/ $\mathrm{Fe}_{3} \mathrm{O}_{4}$ composite nanoparticles in tiny pools of water-in-oil microemulsion. Reactive and Functional Polymers, 66, 1552-1558 (2006).

DOI: $10.1016 /$ j.reactfunctpolym.2006.05.006

[15] Liu G., Shao L., Ge F., Chen J.: Preparation of ultrafine chitosan particles by reverse microemulsion. China Particuology, 5, 384-390 (2007).

DOI: $10.1016 /$ j.cpart.2007.08.002
[16] Hamidi M., Azadi A., Rafiei P.: Hydrogel nanoparticles in drug delivery. Advanced Drug Delivery Reviews, 60, 1638-1649 (2008).

DOI: $10.1016 / j . a d d r .2008 .08 .002$

[17] Gaserod O., Smidsrod O., Skjak-Braek G.: Microcapsules of alginate-chitosan-I: A quantitative study of the interaction between alginate and chitosan. Biomaterials, 19, 1815-1825 (1998).

DOI: $\underline{10.1016 / \mathrm{S} 0142-9612(98) 00073-8}$

[18] Banerjee T., Mitra S., Singh A. K., Sharma R. K., Maitr A.: Preparation, characterization and biodistribution of ultrafine chitosan nanoparticles. International Journal of Pharmaceutics, 243, 93-105 (2002). DOI: $\underline{10.1016 / \mathrm{S} 0378-5173(02) 00267-3}$

[19] Moura M. J., Figueiredo M. M., Gil M. H.: Rheological study of genipin cross-linked chitosan hydrogels. Biomacromolecules, 8, 3823-3829 (2007). DOI: $10.1021 / \mathrm{bm} 700762 \mathrm{w}$

[20] Mi F-L., Sung H-W., Shyu S-S.: Drug release from chitosan-alginate complex beads reinforced by a naturally occurring cross-linking agent. Carbohydrate Polymers, 48, 61-72 (2002). DOI: $\underline{10.1016 / \mathrm{S} 0144-8617(01) 00212-0}$

[21] Zhu A., Dai S., Li L., Zhao F.: Salt effects on aggregation of $O$-carboxymethylchitosan in aqueous solution. Colloids and Surfaces B: Biointerfaces, 47, 20-28 (2006).

DOI: 10.1016/j.colsurfb.2005.11.020 